\title{
DNA methylation genome-wide analysis in remnant and primary gastric cancers
}

\author{
Kiichi Sugimoto ${ }^{1,2} \cdot$ Tomoaki Ito $^{1,3} \cdot$ Alicia Hulbert $^{1} \cdot$ Chen Chen $^{1} \cdot$ Hajime Orita $^{3} \cdot$ Masahiro Maeda $^{4} \cdot$ Hiroshi Moro $^{4}$. \\ Takeo Fukagawa $^{5} \cdot$ Toshikazu Ushijima $^{4} \cdot$ Hitoshi Katai $^{5} \cdot$ Ryo Wada $^{6} \cdot$ Koichi Sato $^{3} \cdot$ Kazuhiro Sakamoto $^{2}$. \\ Wayne $\mathrm{Yu}^{7} \cdot$ Michael Considine $^{8} \cdot$ Leslie Cope $^{8} \cdot$ Malcolm V. Brock $^{1}$
}

Received: 26 December 2018 / Accepted: 3 March 2019 / Published online: 12 March 2019

(c) The International Gastric Cancer Association and The Japanese Gastric Cancer Association 2019

\begin{abstract}
Background Although primary (PGC) and remnant gastric cancers (RGC) both originate from the same gastrointestinal organ, they have very distinct clinicopathological behaviors. We hypothesized that there would be distinct differences in DNA methylation patterns that would occur during carcinogenesis of RGC and PGC, and that the differences in methylation patterns may help identify the primary factor contributing to chronic inflammation in patients with RGC.

Methods We investigated the genome-wide DNA methylation patterns of PGC and RGC tissues from 48 patients using the Infinium HumanMethylation450 Beadchip assay. The results were validated by quantitative methylation-specific PCR (qMSP) in separate, independent cohorts.

Results We found that in our training cohort of 48 patients, the most variable genes from the gastric cancer tissues identified by the Infinium HumanMethylation450 Beadchip clustered the resultant heatmap into high and low methylation groups. On multivariate analysis, PGCs contributed significantly to the high methylation group ( $p=0.004$, OR 12.33), which suggested that the promoter methylation status in PGC is higher than that in RGC. Supporting this conclusion was the finding that in a separate qMSP analysis in a test cohort, the EPB41L3 gene, chosen because of its high $\beta$ value on microarray analysis in the gastric cancer tissues, had significantly higher DNA promoter methylation in cancer tissues in the validation PGC tissues than in RGC.

Conclusions This study demonstrated that promoter methylation status in PGC is higher than in RGC. This result may reflect the effects of the absence of Helicobacter pylori on the reduced DNA methylation in the remnant stomach.
\end{abstract}

Keywords Remnant gastric cancer · DNA methylation · Genome-wide analysis · Tumor suppressor gene $\cdot$ Helicobacter pylori

\section{Introduction}

Gastric cancer is the fifth most common malignancy and the third most frequent cause of cancer-related mortality in the world; it is also the leading cause of cancer-related death in eastern Asia [1,2]. If gastric cancer has not spread outside

Kiichi Sugimoto and Tomoaki Ito contributed equally to this work.

Electronic supplementary material The online version of this article (https://doi.org/10.1007/s10120-019-00949-5) contains supplementary material, which is available to authorized users.

Kiichi Sugimoto

ksugimo@juntendo.ac.jp

Extended author information available on the last page of the article the margins of the surgical resection, gastrectomy remains the only effective treatment for long-term disease eradication [3]. In fact, over the years, there have been numerous surgical advances each resulting in an ever increasingly higher survival rate at 5 years [4]. Moreover, recent advances in systemic chemotherapy have improved postoperative outcomes in advanced cases [5, 6]. Consequently, remnant gastric cancer (RGC), which can be defined as a newly incident malignancy developing in the remnant stomach after a distal gastrectomy, has become an important clinical issue [7, 8]. RGC is extremely rare with the incidence being reportedly about $1-3 \%$ of all patients who underwent a distal gastrectomy $[9,10]$. It is well known that remnant gastritis arises in the remnant stomach in almost all patients after a distal gastrectomy [11-13]. This remnant gastritis may reflect chronic 
inflammation from postoperative duodenogastric bile reflux, reduction of gastric secretion, stasis of food due to postoperative denervation as well as the infection of Helicobacter pylori in the remnant stomach $[13,14]$. These factors, amongst others, have been implicated as causal of remnant gastric cancer [13, 14]. However, little is known about the exact mechanism involved in the etiology of remnant gastric cancer.

In other gastrointestinal cancers, such as Barrett's esophageal cancer, colitis-associated colon cancer and hepatitis $\mathrm{C}$ virus-positive hepatocellular carcinoma, chronic inflammation has been associated with carcinogenesis via aberrant DNA methylation [15]. Similarly, chronic inflammation has been reported to occur in the stomach especially during gastric carcinogenesis involving promoter gene hypermethylation [16-18]. However, the epigenetic events involving carcinogenesis in the remnant stomach during the development of remnant gastric cancer have been largely unexplored [19]. We hypothesized that there would be distinct differences in DNA methylation patterns that would occur during carcinogenesis of RGC and primary gastric cancer (PGC). We retrospectively investigated genome-wide DNA methylation in RGC and compared the genome-wide DNA methylation profile in RGC to that of PGC with the secondary aim of understanding if differences in the methylation pattern can help to discern factors contributing to chronic inflammation in patients with RGC.

\section{Methods}

\section{Definition of RGC and PGC}

Definitions of RGC vary among throughout the literature [20]. According to Shimada et al. [21], RGC is defined as a newly developed cancer in the remnant stomach occurring more than 5 years after a distal gastrectomy irrespective of the reason for the original resection. In contrast, PGC is defined as the first occurrence of a non-metastatic malignancy in the stomach. In this study, RGC and PGC were all derived from distinct individuals.

\section{Study populations}

Microarray analyses were performed on a training cohort of 48 formalin-fixed paraffin-embedded (FFPE) gastric tumor tissues from patients with PGC $(n=35)$ or RGC $(n=13)$ who underwent surgery at the Juntendo University Shizuoka Hospital between 2004 and 2011. We also established a test cohort to test the methylation results of any individual genes identified in our microarray analysis using 87 PGC patients who were treated at the Juntendo University Shizuoka Hospital between 2006 and 2012, and 39 RGC patients who were treated at the National Cancer Center Hospital between 2002 and 2012. Annotated clinical data from each patient were obtained from institutional databases and medical records. The study was approved by the Institutional Review Board at both the Juntendo University Shizuoka Hospital and the National Cancer Center Hospital.

\section{Clinicopathological analyses}

All specimens were examined in the following manner [22]: after resection of the stomach, the excised specimen was opened along the greater curvature by the surgeon. Following formalin fixation, the specimen and lymph nodes were examined by a pathologist and clinicopathological factors, i.e., age, gender (male/female), tumor location (upper third; proximal/middle or lower third; distal), histological classification (Japanese classification of gastric carcinoma, 3rd English edition [22]: papillary- or tubular-adenocarcinoma/ poorly differentiated or signet-ring cell carcinoma or mucinous adenocarcinoma), depth of tumor invasion (Japanese classification of gastric carcinoma, 3rd English edition [22]: T1-T3/T4), lymph node metastasis (Japanese classification of gastric carcinoma, 3rd English edition [22]: N0, 1/N2, 3), stage (Japanese classification of gastric carcinoma, 3rd English edition [22]: I, II/III) were recorded. To detect Helicobacter pylori in gastric tissues, standard histological sections were stained with modified Giemsa [23]. Briefly, paraffinembedded sections were routinely dewaxed, immersed in water and then incubated in $2 \%$ Giemsa solution in distilled water for $30 \mathrm{~min}$ at room temperature. After rinsing, the sections were quickly dehydrated with ethanol before being cleaned with xylene and mounted in DPX. A tumor section was considered Helicobacter pylori-positive if Helicobacter pylori was identified by its pathognomonic curved or spiral form and observed beneath or within the mucosal layer overlying the gastric epithelium. To identify Epstein-Barr virus (EBV) in gastric cancer cells, EBV-encoded RNA-1 (EBER1) DNP probes (Roche) were used for in situ hybridization. Briefly, $4 \mu \mathrm{m}$ of positively charged sections from FFPE samples was used [24]. A tumor section was considered EBVpositive if dark brown staining was restricted to the nucleus of the malignant epithelial cells, and EBV negative if staining was undetected or only localized to the benign-appearing lymphoid cells.

\section{Genomic DNA extraction and bisulfite treatment}

Genomic DNA extraction was done as described previously $[25,26]$. FFPE sections were deparaffinized in xylene and washed twice with $100 \%$ ethanol. The tumor area most suitable for macrodissection was identified by a qualified pathologist and recorded by directly marking the representative H\&E stained section. Macrodissection of gastric cancer 
tissue was performed on three to five $10 \mu \mathrm{m}$ unstained sections based on the marked H\&E stained section. The macrodissected tissue sections were digested for $4 \mathrm{~h}$ with proteinase $\mathrm{K}$ (Invitrogen, Carlsbad, CA, USA). The resulting DNA was bisulfite converted using reagents contained within the EZ DNA Methylation Kit (Zymo Research, Irvine, CA, USA) and samples were processed according to the manufacturer's instructions.

\section{Infinium HumanMethylation450 Beadchip Assay}

Our training cohort consisted of 35 PGC samples and 13 RGC samples which were analyzed using the Infinium HumanMethylation450 Beadchip assay which allows the assessment of the methylation status of over $450,000 \mathrm{CpGs}$ throughout the genome. The Infinium HumanMethylation450 Beadchip assay (Illumina, San Diego, CA, USA) was performed in the Johns Hopkins University DNA Microarray Core according to the manufacturer's instructions specific for formalin-fixed embedded material [27]. Locus methylation was calculated as a $\beta$ value using GenomeStudio software with the low to high ranging from 0 to 1 , respectively. Data from samples assayed on the HumanMethylation $450 \mathrm{~K}$ platform were imported into the $\mathrm{R}$ environment $(\mathrm{R}$ Programming Software version 2.15.1) and processed using the Minfi package version 1.10.1, as previously described [28]. The columns of the heatmap were ordered by unsupervised clustering, whereas rows were ordered top-to-bottom by decreasing value of significance for the $t$ statistic for differential methylation. The heat map $Z$ score hierarchical unsupervised clustering was performed using the 50 most differentially methylated probes from the Infinium HumanMethylation450 Beadchip assay that mapped to the promoter regions. Then, the resultant heatmap was divided into two groups, i.e., high and low methylation group based on the first differentiator of the unsupervised clustering. Finally, to perform a validation using quantitative methylation-specific PCR (qMSP), four tumor suppressor genes were chosen (two of these genes were predicted to be highly methylated in PGC, and the another two were predicted to be highly methylated in RGC) based on the criteria of having larger $\beta$ values, being related to $\mathrm{CpG}$ islands in the promoter region, and being suitable for primer and probe design for quantitative methylation-specific PCR (Supplemental Table 1).

\section{Quantitative methylation-specific PCR (qMSP)}

We analyzed the methylation status of the promoter region in each gene in the qMSP analysis. qMSP was performed as follows. Briefly, the bisulfite-modified DNA served as a template for fluorescence-based real-time PCR. qMSP was performed using an ABI StepOnePlus Real-Time PCR System (Applied BioSystems, Waltham, MA, USA). The bisulfite-modified DNA was assessed for target gene promoter methylation with $200 \mathrm{nM}$ forward primer, $200 \mathrm{nM}$ reverse primer and $80 \mathrm{nM}$ probes. Cycling conditions were $95{ }^{\circ} \mathrm{C}$ for $5 \mathrm{~min}$, followed by 55 cycles of $\left(95{ }^{\circ} \mathrm{C}\right.$ for $15 \mathrm{~s}$, $60{ }^{\circ} \mathrm{C}$ for $1 \mathrm{~min}$ and $72{ }^{\circ} \mathrm{C}$ for $1 \mathrm{~min}$ ). The master mix contained $16.6 \mathrm{mM}\left(\mathrm{NH}_{4}\right)_{2} \mathrm{SO}_{4}, 67 \mathrm{mM}$ Tris $\mathrm{pH} 8.8,10 \mathrm{mM}$ $\beta$-mercaptoethanol, $10 \mathrm{nM}$ fluorescein, $0.166 \mathrm{mM}$ of each deoxynucleotide triphosphate (dNTP) and $0.04 \mathrm{U} / \mu \mathrm{l}$ of Platinum Taq polymerase (ThermoFisher Scientific, Waltham, MA, USA). Final reaction volumes for all assays were $25 \mu$. As a positive methylation control, we utilized Human female Jurkat genomic DNA treated with CpG Methylase (M.SssI) (New England Bio Labs, Ipswich, MA, USA). With the extremely low levels of DNA methylation in samples, replicates for some samples produced no detectable methylation as expected. For the final quantification of methylation (relative methylation value: RMV), we calculated the $2^{-\Delta \Delta \mathrm{Ct}}$ for each methylation detection replicate comparing it to the mean Ct for B-ACTIN (ACTB) [29]. For replicates which were not detected, a $\mathrm{Ct}$ of 100 was used, creating a near zero value for $2^{-\Delta \Delta \mathrm{Ct}}$. The mean $2^{-\Delta \Delta \mathrm{Ct}}$ value (RMV) was calculated with this formula: the mean $2^{-\Delta \Delta \mathrm{Ct}}(\mathrm{RMV})=\left(2^{-\Delta \Delta \mathrm{Ct}}\right.$ replicate_1 $\left.+2^{-\Delta \Delta \text { Ct_replicate_2 }}+2^{-\Delta \Delta \text { Ct_replicate_3 }}\right) / 3$ [29].

\section{Statistical analysis}

The Fisher's exact probability test was employed to compare discrete variables. Continuous variables were compared using the Mann-Whitney $U$ test for individual comparisons and the Wilcoxon signed rank test for paired comparisons. Clinicopathological factors, for which there were significant differences in the univariate analysis, were used as covariables for the multivariate analysis. For the multivariate analysis, the logistic regression model was used with the odds ratio (OR) and $95 \%$ confidence intervals as a measure of association by applying a stepwise procedure. Data were analyzed statistically using JMP 9 software (SAS Institute Inc., Cary, NC, USA). Differences were considered statistically significant at $p<0.05$. Values are expressed as the median (range).

\section{Results}

\section{Comparisons of the clinicopathological factors between PGC and RGC in the training cohort}

The patient characteristics in the training cohort are shown in Table 1. By univariate analysis, there was a significant difference in the tumor location between the two groups $(p=0.0005)$ as well as a trend towards more patients with stage I-II $(p=0.052)$ and Epstein-Barr virus infection $(p=0.06)$ in the RGC group. There were no significant univariate differences 
Table 1 Comparisons of the clinicopathological factors between primary gastric cancer (PGC) and remnant gastric cancer (RGC) in the training cohort

\begin{tabular}{|c|c|c|c|c|}
\hline Clinicopathological factors & Variables & PGC $(n=35)$ & $\operatorname{RGC}(n=13)$ & $p$ value \\
\hline Age & & $72(52-89)^{\mathrm{d}}$ & $75(62-84)^{\mathrm{d}}$ & 0.16 \\
\hline \multirow[t]{2}{*}{ Gender } & Male & $27(77.1 \%)$ & $11(84.6 \%)$ & \multirow[t]{2}{*}{0.71} \\
\hline & Female & $8(22.9 \%)$ & $2(15.4 \%)$ & \\
\hline \multirow[t]{2}{*}{ Tumor location ${ }^{\mathrm{a}}$} & Proximal & $16(45.7 \%)$ & $13(100 \%)$ & \multirow[t]{2}{*}{0.0005} \\
\hline & Distal & $19(54.3 \%)$ & $0(0 \%)$ & \\
\hline Interval between surgeries (range) & & - & 20 years $(8.4-48)$ & - \\
\hline \multirow[t]{2}{*}{ Indication for initial surgery } & Ulcer & - & $8(61.5 \%)$ & \multirow[t]{2}{*}{-} \\
\hline & Cancer & & $5(38.5 \%)$ & \\
\hline \multirow[t]{2}{*}{ Surgical reconstructions } & Billroth-I & - & $10(76.9 \%)$ & \multirow[t]{2}{*}{-} \\
\hline & Billroth-II & & $3(23.1 \%)$ & \\
\hline \multirow[t]{2}{*}{ Histological classification $^{\mathrm{b}}$} & Differentiated & $21(60.0 \%)$ & $6(46.2 \%)$ & \multirow[t]{2}{*}{0.52} \\
\hline & Undifferentiated & $14(40.0 \%)$ & $7(53.8 \%)$ & \\
\hline \multirow[t]{2}{*}{ Depth of tumor invasion ${ }^{c}$} & $\mathrm{~T} 1, \mathrm{~T} 2$ & $12(34.3 \%)$ & $8(61.5 \%)$ & \multirow[t]{2}{*}{0.11} \\
\hline & $\mathrm{T} 3, \mathrm{~T} 4$ & $23(65.7 \%)$ & $5(38.5 \%)$ & \\
\hline \multirow[t]{2}{*}{ Lymph node metastasis ${ }^{\mathrm{c}}$} & N0, N1 & $17(48.6 \%)$ & $10(76.9 \%)$ & \multirow[t]{2}{*}{0.11} \\
\hline & $\mathrm{N} 2, \mathrm{~N} 3$ & $18(51.4 \%)$ & $3(23.1 \%)$ & \\
\hline \multirow[t]{2}{*}{ Stage $^{c}$} & I, II & $15(42.9 \%)$ & $10(76.9 \%)$ & \multirow[t]{2}{*}{0.052} \\
\hline & III & $20(57.1 \%)$ & $3(23.1 \%)$ & \\
\hline \multirow[t]{2}{*}{ Helicobacter pylori } & Positive & $12(34.3 \%)$ & $3(23.1 \%)$ & \multirow[t]{2}{*}{0.73} \\
\hline & Negative & $23(65.7 \%)$ & $10(76.9 \%)$ & \\
\hline \multirow[t]{2}{*}{ Epstein-Barr virus } & Positive & $1(2.9 \%)$ & $3(23.1 \%)$ & \multirow[t]{2}{*}{0.06} \\
\hline & Negative & $34(97.1 \%)$ & $10(76.9 \%)$ & \\
\hline
\end{tabular}

$P G C$ primary gastric cancer, $R G C$ remnant gastric cancer

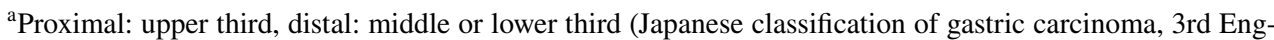
lish edition [22])

${ }^{\mathrm{b}}$ Differentiated: papillary- or tubular-adenocarcinoma, Undifferentiated: poorly differentiated or signet-ring cell carcinoma or mucinous adenocarcinoma (Japanese classification of gastric carcinoma, 3rd English edition [22])

${ }^{\mathrm{c} J a p a n e s e}$ classification of gastric carcinoma, 3rd English edition [22]

${ }^{\mathrm{d}}$ Median (range) between the two groups with respect to other clinicopathological factors. Although the rate of Helicobacter pylori infection in the PGC group was higher than in the RGC group $34.3 \%$ vs. $23.1 \%$, respectively), there was no significant univariate difference in the prevalence of Helicobacter pylori between the two groups $(p=0.73)$. In the RGC group, the median interval between the first and second operations was 20 years (8.4-48 years). The indications for the initial surgery were gastric/duodenum ulcer in eight patients and gastric cancer in five patients. There were ten patients with Billroth-I (B-I) reconstructions and three patients with Billroth-II (B-II) reconstructions (Table 1). In the final molecular analysis, two patients (one PGC patient and one RGC patient) were excluded due to insufficient DNA quality, leaving 46 patients for inclusion in the study.

\section{Using DNA methylation of the most variable tumor suppressor genes to cluster samples in the training cohort}

We clustered samples by DNA promoter hypermethylation according to the most variable tumor suppressor genes observed. Then, we divided the resultant heatmap into two groups, i.e., high and low methylation groups as described in the "Methods" (Fig. 1). The high and the low methylation groups differed in clinicopathological factors related to an aggressive malignant phenotype (Table 2). In univariate analysis, there were significant differences between the high and the low methylation groups in the type of cancer $(p=0.001)$, invasion depth $(p=0.03)$, lymph node metastasis $(p=0.005)$ and advanced cancer stage $(p=0.01)$. 
Fig. 1 Clustering of samples by DNA promoter hypermethylation of the most variable tumor suppressor genes. Heat map $Z$ score hierarchical clustering from Illumina Infinium HumanMethylation450 Beadchip array of the 50 most differentially methylated probes mapping the promoter regions in 46 human gastric tissue DNA samples. Each row represents one probe and each column represents one sample. Only probes within $1500 \mathrm{bp}$ of the transcription start site were considered since this area was used as a rough determination of the promoter region. Samples were arranged left to right and then the heatmap was divided into high and low methylation groups based on the first differentiator of the unsupervised clustering. The level of DNA methylation ( $\beta$ value) for each probe, in each sample, is represented using a color scale as shown in the legend

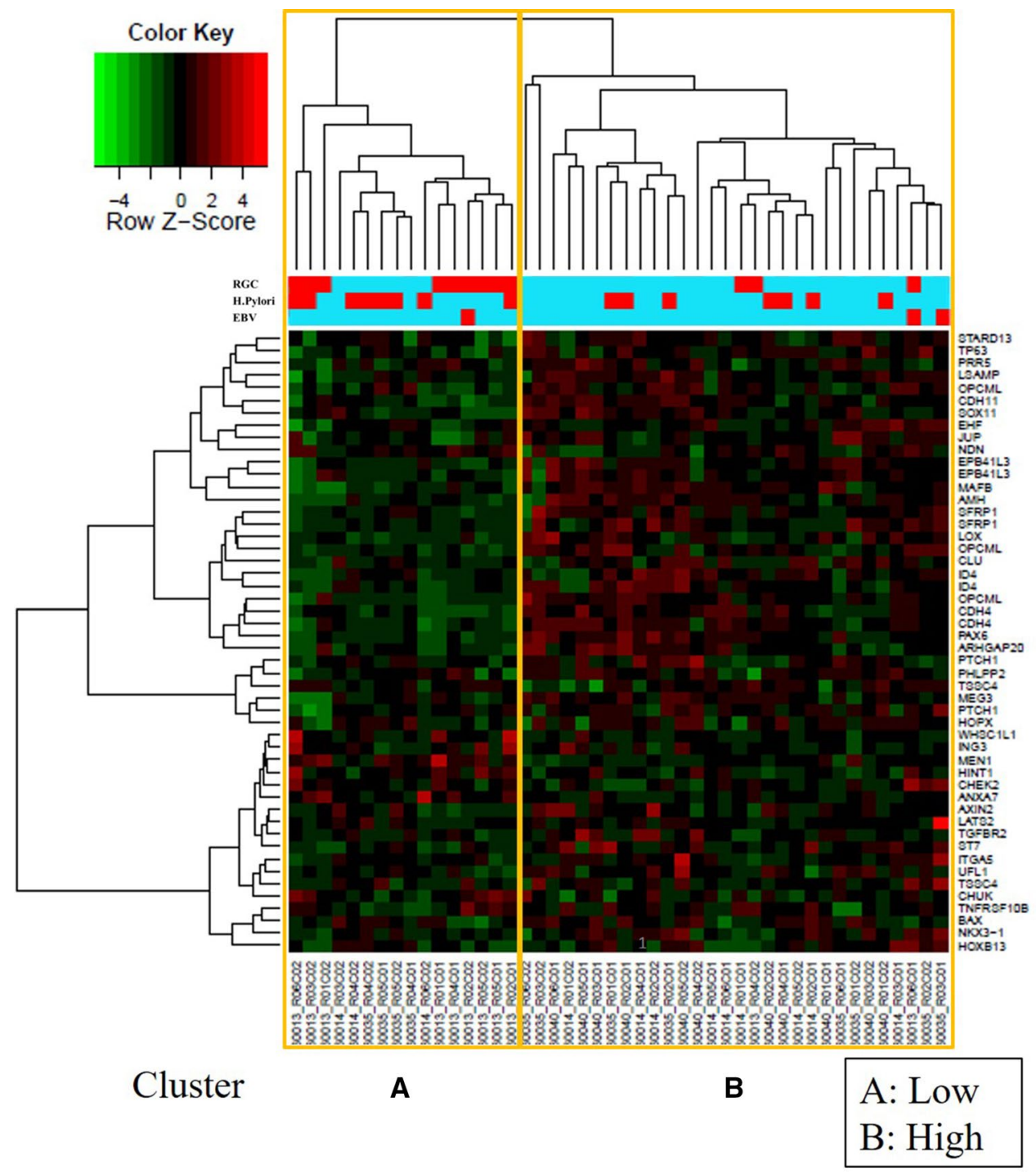

Specifically, patients in the high methylation group were more likely to have PGC, an invasion depth of T3, 4, lymph node metastases (N2, 3) and/or stage III disease. There were no significant differences observed between groups with respect to other clinicopathological factors. Similarly, there were no significant univariate differences between the two groups with respect to Helicobacter pylori $(p=0.10)$ and Epstein-Barr virus infections $(p=1.00)$. When these clinicopathological factors were used as co-variables for a multivariate analysis, the type of cancer (PGC) was found to be a significant independent predictive factor $(p=0.004$, OR 12.33) (Table 3). Finally, we selected four genes which were found in our microarray analysis to be strongly associated with codifying our training set into high and low methylation groups. Two of these genes were predicted to be highly methylated in PGC (EPB41L3 [30] and SOX11 [31]) and another two were predicted to be highly methylated in RGC (TNFRSF10B and ING3) (Supplemental Table 1).

\section{Comparisons of the clinicopathological factors between patients with PGC and RGC in the test cohort}

The demographics of the patients in the test cohort are shown in Table 4. By univariate analysis, there were significant differences in gender $(p=0.006)$, tumor location $(p<0.001)$ and invasion depth $(p=0.02)$ between the two groups. There were no significant univariate differences between the two groups, however, with respect to other clinicopathological factors. In the RGC group, the median interval between the first and second operations was 28 years (6-53 years). There were 21 patients with a Billroth-I (B-I) reconstructions and 18 patients with a Billroth-II (B-II) reconstructions. 
Table 2 Differences

in clinicopathological characteristics of high and low methylation groups in the clustering of samples by the top 50 most variable probes in the promoter regions using the Infinium HumanMethylation450 Beadchip Assay

\begin{tabular}{|c|c|c|c|c|}
\hline Clinicopathological factors & Variables & $\begin{array}{l}\text { High methylation } \\
\text { group }^{\mathrm{a}}(n=30)\end{array}$ & $\begin{array}{l}\text { Low methylation } \\
\text { group }^{\mathrm{a}}(n=16)\end{array}$ & $p$ value \\
\hline Age & & $74(52-89)^{\mathrm{f}}$ & $74.5(56-84)^{\mathrm{f}}$ & 0.98 \\
\hline \multirow[t]{2}{*}{ Gender } & Male & $24(80.0 \%)$ & $13(81.3 \%)$ & 1.00 \\
\hline & Female & $6(20.0 \%)$ & $3(18.7 \%)$ & \\
\hline \multirow[t]{2}{*}{ Tumor location $^{\mathrm{b}}$} & Proximal & $18(60.0 \%)$ & $10(62.5 \%)$ & 1.00 \\
\hline & Distal & $12(40.0 \%)$ & $6(37.5 \%)$ & \\
\hline \multirow[t]{2}{*}{ Histological classification $^{\mathrm{c}}$} & Differentiated & $17(56.7 \%)$ & $9(56.3 \%)$ & 1.00 \\
\hline & Undifferentiated & $13(43.3 \%)$ & $7(43.7 \%)$ & \\
\hline \multirow[t]{2}{*}{ Type of gastric cancer ${ }^{\mathrm{d}}$} & PGC & $27(90.0 \%)$ & $7(43.7 \%)$ & 0.001 \\
\hline & RGC & $3(10.0 \%)$ & $9(56.3 \%)$ & \\
\hline \multirow[t]{2}{*}{ Depth of tumor invasion ${ }^{\mathrm{e}}$} & $\mathrm{T} 1, \mathrm{~T} 2$ & $8(26.7 \%)$ & $10(62.5 \%)$ & 0.03 \\
\hline & $\mathrm{T} 3, \mathrm{~T} 4$ & $22(73.3 \%)$ & $6(37.5 \%)$ & \\
\hline \multirow[t]{2}{*}{ Lymph node metastasis $^{\mathrm{e}}$} & N0, N1 & $13(43.3 \%)$ & $14(87.5 \%)$ & 0.005 \\
\hline & $\mathrm{N} 2, \mathrm{~N} 3$ & $17(56.7 \%)$ & $2(12.5 \%)$ & \\
\hline \multirow[t]{2}{*}{ Stage $^{e}$} & I, II & $12(40.0 \%)$ & $13(81.3 \%)$ & 0.01 \\
\hline & III & $18(60.0 \%)$ & $3(18.7 \%)$ & \\
\hline \multirow[t]{2}{*}{ Helicobacter pylori } & Positive & $7(23.3 \%)$ & $8(50.0 \%)$ & 0.10 \\
\hline & Negative & $23(76.7 \%)$ & $8(50.0 \%)$ & \\
\hline \multirow[t]{2}{*}{ Epstein-Barr virus } & Positive & $2(6.7 \%)$ & $1(6.3 \%)$ & 1.00 \\
\hline & Negative & $28(93.3 \%)$ & $15(93.7 \%)$ & \\
\hline
\end{tabular}

${ }^{a}$ The resultant heatmap was divided into two groups, i.e., high and low methylation group based on the first differentiator of the unsupervised clustering by the top 50 most variable probes in the promoter regions (Fig. 1)

${ }^{\text {b} P r o x i m a l: ~ u p p e r ~ t h i r d, ~ d i s t a l: ~ m i d d l e ~ o r ~ l o w e r ~ t h i r d ~(J a p a n e s e ~ c l a s s i f i c a t i o n ~ o f ~ g a s t r i c ~ c a r c i n o m a, ~ 3 r d ~ E n g-~}$ lish edition [22])

${ }^{c}$ Differentiated: papillary- or tubular-adenocarcinoma, Undifferentiated: poorly differentiated or signet-ring cell carcinoma or mucinous adenocarcinoma (Japanese classification of gastric carcinoma, 3rd English edition [22])

${ }^{\mathrm{d}}$ PGC: primary gastric cancer, RGC: remnant gastric cancer

$\mathrm{e} J a p a n e s e$ classification of gastric carcinoma, 3rd English edition [22]

${ }^{\mathrm{f}}$ Median (range)

\begin{tabular}{lllcl}
\hline Clinicopathological factors & Variable & $p$ value & Odds ratio & 95\% confidence interval \\
\hline Type of gastric cancer & PGC $^{\mathrm{a}}$ & 0.004 & 12.33 & $2.14-109.10$ \\
Depth of tumor invasion & $\mathrm{T} 3,4^{\mathrm{b}}$ & 0.05 & 8.35 & $0.98-110.07$ \\
Lymph node metastasis & $\mathrm{N} 2,3^{\mathrm{b}}$ & 0.10 & 17.67 & $0.51-700.46$ \\
Stage & III $^{\mathrm{b}}$ & 0.18 & 0.08 & $0.00-3.68$
\end{tabular}

The resultant heatmap was divided into two groups, i.e., high and low methylation group based on the first differentiator of the unsupervised clustering by the top 50 most variable probes in the promoter regions (Fig. 1)

a PGC: primary gastric cancer, RGC: remnant gastric cancer

${ }^{\mathrm{b}}$ Japanese classification of gastric carcinoma, 3rd English edition [22]

\section{Quantitative methylation-specific PCR (qMSP) in the test cohort}

Using qMSP, we sought to validate the results of our microarray analysis in four genes in the test cohort. First, we compared the RMVs of four genes in PGC and normal-appearing adjacent non-cancerous gastric tissues (Supplemental Fig. 1). For the gene TNFRSF10B, RMVs in PGC cancer tissues were significantly lower than those in normal-appearing adjacent non-cancerous tissues $(p=0.003)$. There were no significant differences in RMVs between cancer tissues and normal-appearing adjacent non-cancerous tissues for the 
Table 4 Differences in clinicopathological factors between primary gastric cancer (PGC) and remnant gastric cancer (RGC) in the test cohort

\begin{tabular}{|c|c|c|c|c|}
\hline Clinicopathological factors & Variables & PGC $(n=87)$ & $\operatorname{RGC}(n=39)$ & $p$ value \\
\hline Age & & $71(33-86)^{\mathrm{d}}$ & $70(37-84)^{\mathrm{d}}$ & 0.48 \\
\hline \multirow[t]{2}{*}{ Gender } & Male & $60(69.0 \%)$ & $36(92.3 \%)$ & \multirow[t]{2}{*}{0.006} \\
\hline & Female & $27(31.0 \%)$ & $3(7.7 \%)$ & \\
\hline \multirow[t]{2}{*}{ Tumor location $^{\mathrm{a}}$} & Proximal & $30(34.5 \%)$ & $39(100 \%)$ & \multirow[t]{2}{*}{$<0.001$} \\
\hline & Distal & $57(65.5 \%)$ & $0(0 \%)$ & \\
\hline Interval between surgeries (range) & & - & 28 years $(6-53)$ & - \\
\hline \multirow[t]{2}{*}{ Surgical reconstructions } & Billroth-I & - & $21(53.8 \%)$ & \multirow[t]{2}{*}{-} \\
\hline & Billroth-II & & $18(46.2 \%)$ & \\
\hline \multirow[t]{2}{*}{ Histological classification $^{\mathrm{b}}$} & Differentiated & $46(52.9 \%)$ & $21(53.8 \%)$ & \multirow[t]{2}{*}{1.00} \\
\hline & Undifferentiated & $41(47.1 \%)$ & $18(46.2 \%)$ & \\
\hline \multirow[t]{2}{*}{ Depth of tumor invasion ${ }^{c}$} & $\mathrm{~T} 1, \mathrm{~T} 2$ & $50(57.5 \%)$ & $13(33.3 \%)$ & \multirow[t]{2}{*}{0.02} \\
\hline & $\mathrm{T} 3, \mathrm{~T} 4$ & $37(42.5 \%)$ & $26(66.7 \%)$ & \\
\hline \multirow[t]{2}{*}{ Lymph node metastasis $^{c}$} & N0, N1 & $63(72.4 \%)$ & $32(82.1 \%)$ & \multirow[t]{2}{*}{0.27} \\
\hline & $\mathrm{N} 2, \mathrm{~N} 3$ & $24(27.6 \%)$ & $7(17.9 \%)$ & \\
\hline \multirow[t]{2}{*}{ Stage $^{c}$} & I, II & $60(69.0 \%)$ & $30(76.9 \%)$ & \multirow[t]{2}{*}{0.40} \\
\hline & III & $27(31.0 \%)$ & $9(23.1 \%)$ & \\
\hline
\end{tabular}

$P G C$ primary gastric cancer, $R G C$ remnant gastric cancer

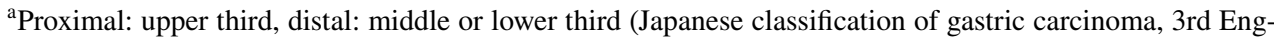
lish edition [22])

${ }^{\mathrm{b}}$ Differentiated: papillary- or tubular-adenocarcinoma, Undifferentiated: poorly differentiated or signet-ring cell carcinoma or mucinous adenocarcinoma (Japanese classification of gastric carcinoma, 3rd English edition [22])

${ }^{\mathrm{c} J a p a n e s e}$ classification of gastric carcinoma, 3rd English edition [22]

${ }^{\mathrm{d}}$ Median (range)

other three genes examined (EPB41L3, SOX11 and ING3). Second, we compared the RMVs of the four genes in RGC and normal-appearing adjacent non-cancerous gastric tissues (Supplemental Fig. 2). For three genes (EPB41L3, SOX11 and ING3), RMVs in RGC cancer tissues were significantly higher than those in normal-appearing adjacent non-cancerous tissues ( $p<0.001, p<0.001$ and $p=0.008$, respectively). But, for TNFRSF10B, the RMV in RGC was significantly lower than those in normal-appearing adjacent non-cancerous gastric tissue $(p=0.01)$. Third, we compared the RMVs of the four genes in cancer tissues between all PGC (PGCtotal), the cases with the distal side of PGC (PGC-distal), the cases with the proximal side of PGC (PGC-proximal) and RGC (Fig. 2). For the EPB41L3 gene, the RMV in PGC-total was significantly higher than in RGC $(p=0.01)$. Similarly, the RMV in PGC-proximal was significantly higher than in RGC $(p=0.003)$. For the TNFRSF10B gene, the RMV in PGC-proximal was significantly higher than in PGC-distal $(p=0.01)$. There were no significant differences in RMVs for the other two genes (SOX11 and ING3). Fourth, we compared the RMVs of the four genes in normal-appearing adjacent non-cancerous tissues between all PGC (NPG-total), the cases with the distal side of PGC (NPG-distal), the cases with the proximal side of PGC (NPG-proximal) and RGC (NRG) (Fig. 3). For three genes (EPB41L3, SOX11 and ING3), the RMVs in NPG-total were significantly higher than in NRG $(p<0.001, p<0.001$ and $p=0.004$, respectively). Similarly, for three genes (EPB41L3, SOX11 and ING3), the RMVs in NPG-distal were significantly higher than in NRG $(p<0.001, p<0.001$ and $p=0.007$, respectively). But, for the TNFRSF10B gene, the RMV in NPGtotal was significantly lower than in NRG $(p=0.02)$. Similarly, the RMV in NPG-distal was significantly lower than in NRG $(p=0.02)$. Finally, we compared the RMVs of the four genes both in cancer tissues and in normal-appearing adjacent non-cancerous gastric tissues of RGC between Billroth-I and -II reconstructions (Supplemental Figs. 3 and 4 , respectively). Consequently, for all of the four genes, there were no significant differences in RMVs in both tissues of RGC between the two reconstructions.

\section{Discussion}

Despite the fact that primary and remnant gastric cancers both are exposed to long-term chronic inflammation in the same gastrointestinal organ, their distinct clinicopathological behaviors suggest separate etiological carcinogenic pathways. We performed genome-wide, DNA promoter hypermethylation analysis using the Infinium HumanMethylation450 Beadchip to examine if the DNA methylation profile between the two diseases was different. We found 

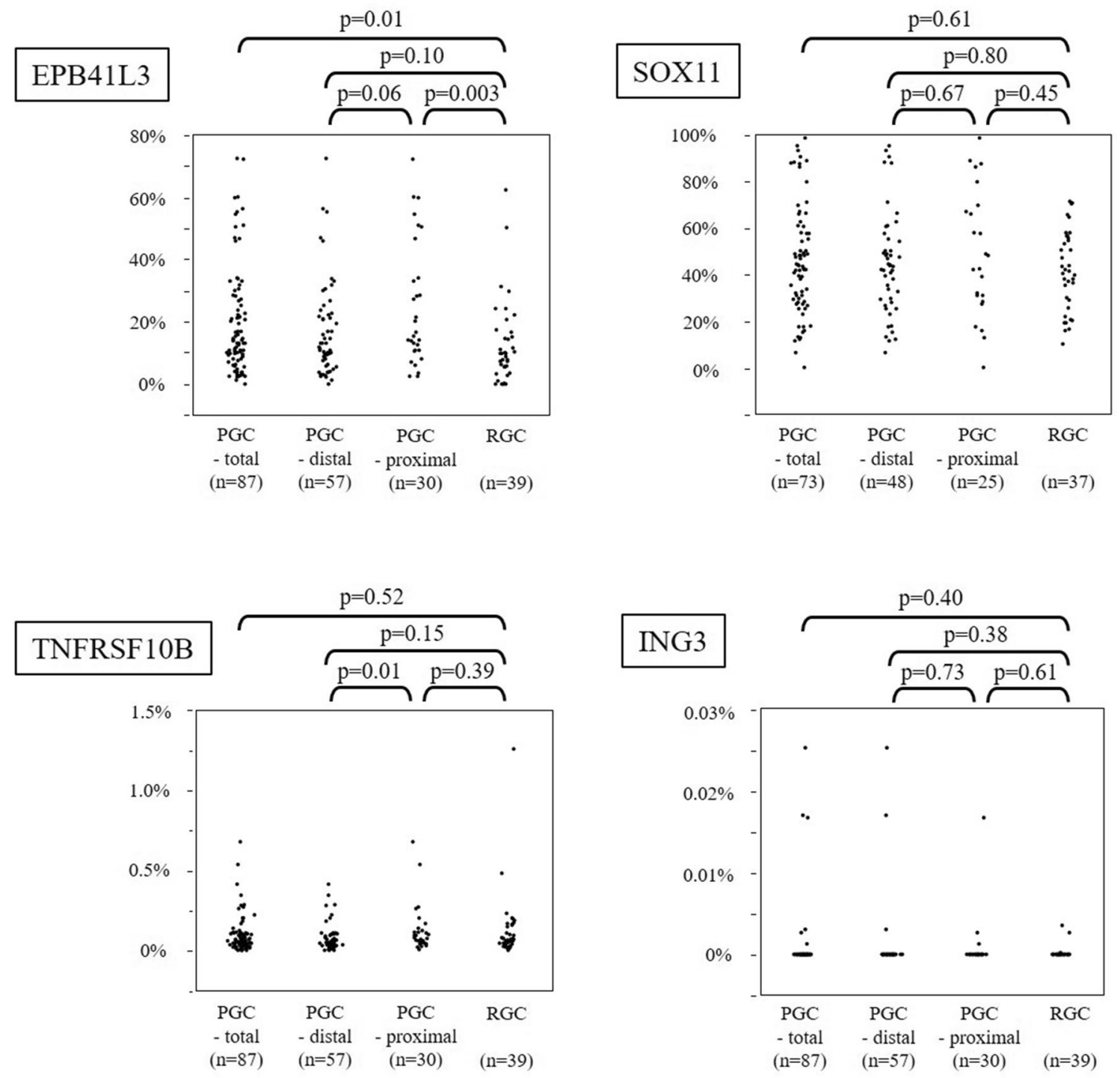

Fig. 2 Relative methylation values (RMVs) comparing four genes in cancer tissues between all primary gastric cancer (PGC-total), the cases with the distal side of PGC (PGC-distal), the cases with the proximal side of PGC (PGC-proximal) and remnant gastric cancer (RGC). RMVs of the four genes in cancer tissues between all PGC (PGC-total), the cases with the distal side of PGC (PGC-distal),

that the resulting genes identified by the Infinium HumanMethylation450 Beadchip clustered into high and low methylation groups on multivariate analysis. PGCs contributed significantly to the high methylation group suggesting that the promoter methylation status in PGC is higher than that in $\operatorname{RGC~(~} p=0.004$, OR 12.33). Supporting this conclusion is our finding that in a separate qMSP analysis in a test cohort, the EPB41L3 gene, chosen because of its suggested hypermethylation in PGC (its high $\beta$ value on microarray analysis), had significantly higher DNA promoter methylation in cancer tissues in PGC than in RGC. Three other genes also the cases with the proximal side of PGC (PGC-proximal) and RGC were compared. For the EPB41L3 gene, the RMV in PGC-total was significantly higher than in RGC $(p=0.01)$. Similarly, the RMV in PGC-proximal was significantly higher than in $\operatorname{RGC}(p=0.003)$. For the TNFRSF10B gene, the RMV in PGC-proximal was significantly higher than in PGC-distal $(p=0.01)$

with high $\beta$ values on microarray analysis did not demonstrate a significant difference between the two groups.

It is well known that DNA promoter hypermethylation plays a crucial role in the carcinogenesis of tumors in the stomach [32,33]. In particular, Helicobacter pylori infection is known to trigger inflammatory processes which can cause aberrant DNA methylation in gastric epithelial cells [34]. It is possible that, with the concomitant loss of the antrum and exposure to bile reflux, the resultant alkalinization of the remnant stomach may induce the elimination of Helicobacter pylori there since these bacteria can only survive in 

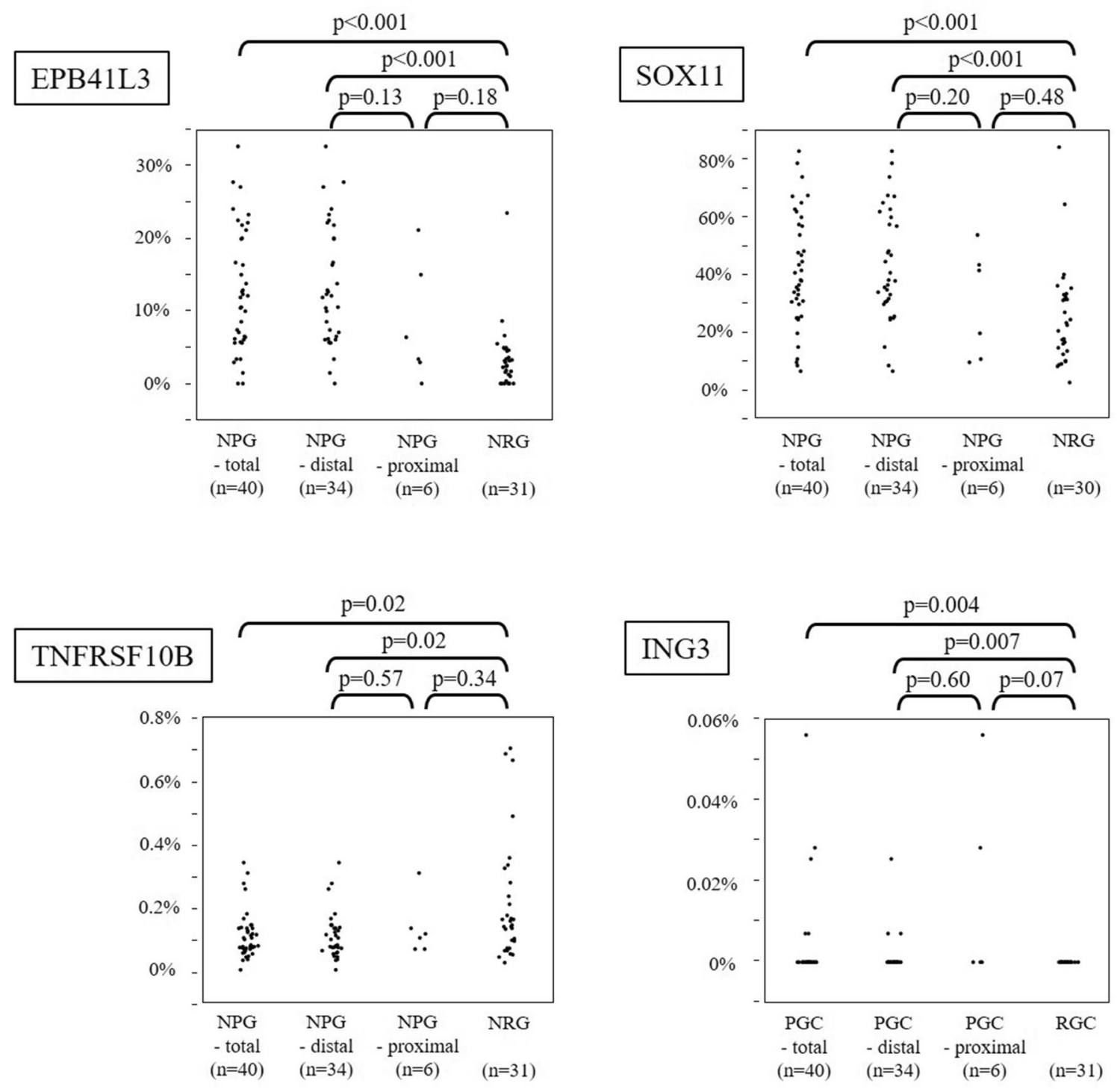

Fig. 3 Relative methylation values (RMVs) comparing four genes in normal-appearing adjacent non-cancerous tissues between all primary gastric cancer (NPG-total), the cases with the distal side of PGC (NPG-distal), the cases with the proximal side of PGC (NPGproximal) and remnant gastric cancer (NRG). RMVs of the four genes in normal-appearing adjacent non-cancerous tissues between all PGC (NPG-total), the cases with the distal side of PGC (NPGdistal), the cases with the proximal side of PGC (NPG-proximal) and RGC (NRG) were compared. For three genes (EPB41L3, SOX11

an acidotic environment. Bile acids are also known to have inhibitory effects on the adherence and growth of Helicobacter pylori in vitro $[35,36]$. In fact, there are multiple reports that the prevalence of Helicobacter pylori infection is significantly lower in patients with severe duodenogastric bile reflux than in those without duodenogastric bile reflux [37-39]. Helicobacter pylori has also been reported to be a very strong inducer of DNA methyltransferase [32, 33]. Helicobacter pylori-induced chronic inflammation, but and ING3), the RMVs in NPG-total were significantly higher than in NRG $(p<0.001, p<0.001$ and $p=0.004$, respectively). Similarly, for three genes (EPB41L3, SOX11 and ING3), the RMVs in NPGdistal were significantly higher than in NRG $(p<0.001, p<0.001$ and $p=0.007$, respectively). But, for the TNFRSF10B gene, the RMV in NPG-total was significantly lower than in NRG $(p=0.02)$. Similarly, the RMV in NPG-distal were significantly lower than in NRG $(p=0.02)$

not Helicobacter pylori itself, may also play a direct role in the induction of aberrant DNA methylation [33]. Moreover, aberrant DNA methylation has not been shown to be induced by chemicals such as high concentrations of alcohol or saturated $\mathrm{NaCl}$ [34]. Therefore, it is possible that our observation that the methylation status in RGC may be reduced is due to the absence of adherent Helicobacter pylori, irrespective of the physical stimulation of the bile reflux to the mucosa of the remnant stomach or the chronic mucosal inflammation 
from bile reflux and subsequent induction of superoxide as well as nitric oxide which are well-known inducers of DNA methyltransferase [40, 41]. Consistent with these observations, there were no significant differences in RMVs both in cancer tissues and in normal-appearing adjacent noncancerous gastric tissues of RGC between Billroth-I and -II reconstructions. This may indicate that bile reflux has no immediate effect on the induction of DNA methylation on the mucosa in the remnant stomach because the Billroth-II reconstruction is usually thought to cause bile reflux more than the Billroth-I reconstruction due to the anatomical structure. Indeed, Lee et al. [42] reported that there is no correlation between histopathologically evident chronic inflammation in the remnant stomach and bile reflux or Helicobacter pylori infection. In addition, Maeda et al. [33] stated that aberrant DNA methylation can even be present in non-cancerous gastric mucosa, and its level is influenced by Helicobacter pylori infection. Our findings also seem to suggest that the RMVs of three genes (EPB41L3, SOX11 and ING3) in normal-appearing adjacent non-cancerous tissues in PGC were significantly higher than in RGC. These observations are in concordance with the findings of Maeda et al. [33]. However, we could not demonstrate that the rate of the infection of Helicobacter pylori in the PGC group was significantly higher than in the RGC group (34.3\% and $23.1 \%$, respectively) (Table 1). The reason for this may be due to the fact that only Giemsa staining was employed to detect Helicobacter pylori in gastric tissues. Moreover, almost all gastric cancers develop in persons infected with Helicobacter pylori and not in uninfected persons [43]. Moreover, in general, the sensitivity of Giemsa staining is reportedly lower compared to immunohistochemistry or fluorescent in situ hybridization [44] which theoretically could increase the false-negative readings for Helicobacter pylori in the PGC group in our study. This might have affected our results in comparing the clinicopathological characteristics between high and low methylation groups (Table 2). Moreover, since gastric cancer has recently been suggested to be associated with other bacterial compositions other than Helicobacter pylori, other bacterial flora may affect the methylation status in PGC [45]. Finally, our lack of data regarding Helicobacter pylori infection in our test cohort, limits our ability to clarify this issue.

There have been two reports about DNA gene methylation in RGC. Nakase et al. [17] investigated the RUNX 3 gene methylation in RGC patients with qualitative MSP. They stated that the rate of methylated RUNX3 in cancer tissues was higher than that in non-cancerous adjacent tissues and concluded that the detection of RUNX3 gene methylation may estimate the forward risk of carcinogenesis in the remnant stomach. Their study demonstrated similar results to ours in terms of higher gene methylation rates in three genes in cancer tissues compared with normal-appearing adjacent non-cancerous tissues. Mino et al. [18] investigated p16 gene methylation of cancer tissues with qualitative MSP in two separate analyses for those RGCs which had newly developed after surgery performed either for benign disease or for gastric cancer. They concluded that p16 gene hypermethylation was found more frequently in RGC which developed after surgery for benign disease than in PGC or in RGC which developed after surgery for gastric cancer. Both previous studies concluded that DNA methylation seemed to be a unique molecular mechanism in RGC. However, they studied only one gene in each report which gives limited insight into DNA hypermethylation across the whole genome. Our results may contradict that of Mino et al. [18] since we found that overall PGC promoter methylation is higher than in RGC. Our findings, however, are corroborated by (1) the RMVs in normal-appearing adjacent non-cancerous tissues in PGC were also significantly higher than those in RGC;+ (2) cancer tissues in RGC demonstrated significantly higher RMVs in three genes (except for TNFRSF10B) than those in normal-appearing adjacent non-cancerous tissues in RGC. However, the RMVs in none of the genes in cancer tissues in RGC were higher than those in PGC, which means that PGC would be more likely to be hypermethylated than RGC under the influence of chronic inflammation from Helicobacter pylori infection. Actually, we compared p16 gene hypermethylation with qMSP between PGC and RGC. Consequently, there were no significant differences in RMVs between the two groups (data not shown). The differences in the results between our study and that of Mino et al. seems to be due to the former being quantitative while the latter is qualitative in nature. Given our microarray analysis and our qPCR validation, we are confident in our results showing a lack of difference between cancers in terms of p16 methylation.

There are several limitations in this study that should be considered. First, only a small number of patients were enrolled in this study because RGC is a rare disease. Second, as previously mentioned, we did not have any data about the infection of Helicobacter pylori and Epstein-Barr virus in the samples from the test cohort. Epstein-Barr virus has been known to undergo frequent aberrant DNA methylation in gastric cancer [46-48]. Third, only one gene, EPB41L3, demonstrated significantly higher RMVs in cancer tissues for PGC compared with RGC (Fig. 2). There were no significant differences in RMVs in the other three genes (SOX11, TNFRSF10B and ING3) between PGC and RGC. We chose four genes to analyze further by qMSP based on the results of the Infinium HumanMethylation450 Beadchip. Since DNA gene methylation is known to occur in a gene-specific manner, and the sensitivity to methylation differs between genes [49], other genes may also discriminate between PGC and RGC. Further research needs to be done to investigate these other genes. 
Finally, the remnant stomach may differ in exposure to bile reflux and inflammation according to the type of surgical anastomosis performed after a distal gastrectomy. Ideally, the inclusion in this study of remnant stomach samples from patients who had received a Roux-en-Y anastomosis would have been interesting to compare because this kind of anastomosis is usually used to prevent bile reflux into the remnant stomach. Given the rarity of RGC, this would be an important group of patients to consider going forward.

In conclusion, this study demonstrated that the promoter methylation status in PGC is higher than in RGC. Our data suggest that this may be due to the effects of the absence of Helicobacter pylori on the reduced DNA methylation in the remnant stomach.

\section{Compliance with ethical standards}

Conflict of interest The authors declare that they have no conflict of interest.

Human rights statement and informed consent: All procedures followed were in accordance with the ethical standards of the responsible committee on human experimentation (institutional and national) and with the Helsinki Declaration of 1964 and later versions. Informed consent to be included in the study, or the equivalent, was obtained from all patients.

\section{References}

1. Torre LA, Bray F, Siegel RL, Ferlay J, Lortet-Tieulent J, Jemal A. Global cancer statistics, 2012. CA Cancer J Clin. 2015;65:87-108.

2. Rahman R, Asombang AW, Ibdah JA. Characteristics of gastric cancer in Asia. World J Gastroenterol. 2014;20:4483-90.

3. Best LM, Mughal M, Gurusamy KS. Laparoscopic versus open gastrectomy for gastric cancer. Cochrane Database Syst Rev. 2016;3:CD011389. https://doi.org/10.1002/14651858.

4. Elimova E, Ajani JA. Surgical resection first for localized gastric adenocarcinoma: are there adjuvant options? J Clin Oncol. 2015;33:3085-91.

5. Lordick F, Lorenzen S, Yamada Y, Ilson D. Optimal chemotherapy for advanced gastric cancer: is there a global consensus? Gastric Cancer. 2014;17:213-25.

6. Ushijima T, Sasako M. Focus on gastric cancer. Cancer Cell. 2004;5:121-5.

7. Ohashi M, Katai H, Fukagawa T, Gotoda T, Sano T, Sasako M. Cancer of the gastric stump following distal gastrectomy for cancer. Br J Surg. 2007;94:92-5.

8. Ohashi M, Morita S, Fukagawa T, Kushima R, Katai H. Surgical treatment of non-early gastric remnant carcinoma developing after distal gastrectomy for gastric cancer. J Surg Oncol. 2015;111:208-12.

9. Furukawa H, Iwanaga T, Hiratsuka M, Imaoka S, Ishikawa O, Kabuto T, et al. Gastric remnant cancer as a metachronous multiple lesion. Br J Surg. 1993;80:54-6.

10. Kodera Y, Yamamura Y, Torii A, Uesaka K, Hirai T, Yasui K, et al. Incidence, diagnosis and significance of multiple gastric cancer. Br J Surg. 1995;82:1540-3.
11. Ritchie WP. Alkaline reflux gastritis: a critical reappraisal. Gut. 1984;25:975-87.

12. Sugiyama Y, Sohma H, Ozawa M, Hada R, Mikami Y, Konn M, et al. Regurgitant bile acids and mucosal injury of the gastric remnant after partial gastrectomy. Am J Surg. 1987;153:399-403.

13. Abe H, Murakami K, Satoh S, Sato R, Kodama M, Arita T, et al. Influence of bile reflux and Helicobacter pylori infection on gastritis in the remnant gastric mucosa after distal gastrectomy. J Gastroenterol. 2005;40:563-9.

14. Kaminishi M. How is it possible to prevent gastric mucosal injury and remnant cancer after distal gastrectomy? J Gastroenterol. 2005;40:661-3.

15. Chiba $\mathrm{T}$, Marusawa $\mathrm{H}$, Ushijima $\mathrm{T}$. Inflammation-associated cancer development in digestive organs: mechanisms and roles for genetic and epigenetic modulation. Gastroenterology. 2012;143:550-63.

16. Maekita T, Nakazawa K, Mihara M, Nakajima T, Yanaoka K, Iguchi M, et al. High levels of aberrant DNA methylation in Helicobacter pylori-infected gastric mucosae and its possible association with gastric cancer risk. Clin Cancer Res. 2006;12:989-95.

17. Nakase Y, Sakakura C, Miyagawa K, Kin S, Fukuda K, Yanagisawa A, et al. Frequent loss of RUNX3 gene expression in remnant stomach cancer and adjacent mucosa with special reference to topography. Br J Cancer. 2005;92:562-9.

18. Mino A, Onoda N, Yashiro M, Aya M, Fujiwara I, Kubo N, et al. Frequent p16 CpG island hypermethylation in primary remnant gastric cancer suggesting an independent carcinogenic pathway. Oncol Rep. 2006;15:615-20.

19. Takeno S, Hashimoto T, Maki K, Shibata R, Shiwaku H, Yamana I, et al. Gastric cancer arising from the remnant stomach after distal gastrectomy: a review. World J Gastroenterol. 2014;20:13734-40.

20. Ohira M, Toyokawa T, Sakurai K, Kubo N, Tanaka H, Muguruma $\mathrm{K}$, et al. Current status in remnant gastric cancer after distal gastrectomy. World J Gastroenterol. 2016;22:2424-33.

21. Shimada H, Fukagawa T, Haga Y, Oba K. Does remnant gastric cancer really differ from primary gastric cancer? A systematic review of the literature by the Task Force of Japanese Gastric Cancer Association. Gastric Cancer. 2016;19:339-49.

22. Japanese Gastric Cancer Association. Japanese classification of gastric carcinoma: 3rd English edition. Gastric Cancer. 2011;14:101-12.

23. Gray SF, Wyatt JI, Rathbone BJ. Simplified techniques for identifying Campylobacter pyloridis. J Clin Pathol. 1986;39:1279.

24. Zhang Y, Chen JN, Dong M, Zhang ZG, Zhang YW, Wu JY, et al. Clinical significance of spasmolytic polypeptide-expressing metaplasia and intestinal metaplasia in EBV-associated and EBVnegative gastric cancer. Hum Pathol. 2017;63:128-38.

25. Brock MV, Hooker CM, Ota-Machida E, Han Y, Guo M, Ames $\mathrm{S}$, et al. DNA methylation markers and early recurrence in stage I lung cancer. N Engl J Med. 2008;358:1118-28.

26. Li Y, Yang Y, Lu Y, Herman JG, Brock MV, Zhao P, et al. Predictive value of CHFR and MLH1 methylation in human gastric cancer. Gastric Cancer. 2015;18:280-7.

27. Wong EM, Joo JE, McLean CA, Baglietto L, English DR, Severi $\mathrm{G}$, et al. Tools for translational epigenetic studies involving formalin-fixed paraffin-embedded human tissue: applying the Infinium HumanMethyation450 Beadchip assay to large population-based studies. BMC Res Notes. 2015;8:543.

28. Fackler MJ, Umbricht CB, Williams D, Argani P, Cruz LA, Merino VF, et al. Genome-wide methylation analysis identifies genes specific to breast cancer hormone receptor status and risk of recurrence. Cancer Res. 2011;71:6195-207.

29. Hulbert A, Jusue-Torres I, Stark A, Chen C, Rodgers K, Lee $\mathrm{B}$, et al. Early detection of lung cancer using DNA promoter 
hypermethylation in plasma and sputum. Clin Cancer Res. 2017;23:1998-2005.

30. Eijsink JJ, Yang N, Lendvai A, Klip HG, Volders HH, Buikema HJ, et al. Detection of cervical neoplasia by DNA methylation analysis in cervico-vaginal lavages, a feasibility study. Gynecol Oncol. 2011;120:280-3.

31. Chung W, Bondaruk J, Jelinek J, Lotan Y, Liang S, Czerniak B, et al. Detection of bladder cancer using novel DNA methylation biomarkers in urine sediments. Cancer Epidemiol Biomarkers Prev. 2011;20:1483-91.

32. Ushijima T, Hattori N. Molecular pathways: involvement of Helicobacter pylori-triggered inflammation in the formation of an epigenetic field defect, and its usefulness as cancer risk and exposure markers. Clin Cancer Res. 2012;18:923-9.

33. Maeda M, Moro H, Ushijima T. Mechanisms for the induction of gastric cancer by Helicobacter pylori infection: aberrant DNA methylation pathway. Gastric Cancer. 2017;20:8-15.

34. Niwa T, Tsukamoto T, Toyoda T, Mori A, Tanaka H, Maekita T, et al. Inflammatory processes triggered by Helicobacter pylori infection cause aberrant DNA methylation in gastric epithelial cells. Cancer Res. 2010;70:1430-40.

35. Tompkins DS, West AP. Campylobacter pylori, acid, and bile. J Clin Pathol. 1987;40:1387.

36. Mathai E, Arora A, Cafferkey M, Keane CT, M'Morain C. The effect of bile acids on the growth and adherence of Helicobacter pylori. Aliment Pharmacol Ther. 1991;5:653-8.

37. O'Connor HJ, Dixon MF, Wyatt JI, Axon AT, Ward DC, Dewar EP, et al. Effect of duodenal ulcer surgery and enterogastric reflux on Campylobacter pyloridis. Lancet. 1986;2:1178-81.

38. Onoda N, Maeda K, Sawada T, Wakasa K, Arakawa T, Chung KH. Prevalence of Helicobacter pylori infection in gastric remnant after distal gastrectomy for primary gastric cancer. Gastric Cancer. 2001;4:87-92.

39. Fukuhara K, Osugi H, Takada N, Takemura M, Lee S, Taguchi S, et al. Duodenogastric reflux eradicates Helicobacter pylori after distal gastrectomy. Hepatogastroenterology. 2004;51:1548-50.

40. Hmadcha A, Bedoya FJ, Sobrino F, Pintado E. Methylationdependent gene silencing induced by interleukin 1beta via nitric oxide production. J Exp Med. 1999;190:1595-604.
41. Ohshima H. Genetic and epigenetic damage induced by reactive nitrogen species: implications in carcinogenesis. Toxicol Lett. 2003;140-141:99-104.

42. Lee Y, Tokunaga A, Tajiri T, Masuda G, Okuda T, Fujita I, et al. Inflammation of the gastric remnant after gastrectomy: mucosal erythema is associated with bile reflux and inflammatory cellular infiltration is associated with Helicobacter pylori infection. $\mathrm{J}$ Gastroenterol. 2004;39:520-6.

43. Uemura N, Okamoto S, Yamamoto S, Matsumura N, Yamaguchi S, Yamakido M, et al. Helicobacter pylori infection and the development of gastric cancer. N Engl J Med. 2001;345:784-9.

44. Kocsmár É, Szirtes I, Kramer Z, Szijártó A, Bene L, Buzás GM, et al. Sensitivity of Helicobacter pylori detection by Giemsa staining is poor in comparison with immunohistochemistry and fluorescent in situ hybridization and strongly depends on inflammatory activity. Helicobacter 2017. https://doi.org/10.1111/hel.12387

45. Ferreira RM, Pereira-Marques J, Pinto-Ribeiro I, Costa JL, Carneiro F, Machado JC, et al. Gastric microbial community profiling reveals a dysbiotic cancer-associated microbiota. Gut. 2018;67:226-36.

46. Kang GH, Lee S, Kim WH, Lee HW, Kim JC, Rhyu MG, et al. Epstein-Barr virus-positive gastric carcinoma demonstrates frequent aberrant methylation of multiple genes and constitutes $\mathrm{CpG}$ island methylator phenotype-positive gastric carcinoma. Am J Pathol. 2002;160:787-94.

47. Matsusaka K, Kaneda A, Nagae G, Ushiku T, Kikuchi Y, Hino $\mathrm{R}$, et al. Classification of Epstein-Barr virus-positive gastric cancers by definition of DNA methylation epigenotypes. Cancer Res. 2011;71:7187-97.

48. Cancer Genome Atlas Research Network. Comprehensive molecular characterization of gastric adenocarcinoma. Nature. 2014;513:202-9.

49. Esteller M, Corn PG, Baylin SB, Herman JG. A gene hypermethylation profile of human cancer. Cancer Res. 2001;61:3225-9.

Publisher's Note Springer Nature remains neutral with regard to jurisdictional claims in published maps and institutional affiliations.

\section{Affiliations}

\section{Kiichi Sugimoto ${ }^{1,2} \cdot$ Tomoaki Ito $^{1,3} \cdot$ Alicia Hulbert $^{1} \cdot$ Chen Chen $^{1} \cdot$ Hajime Orita ${ }^{3}$ - Masahiro Maeda ${ }^{4} \cdot$ Hiroshi Moro $^{4}$. Takeo Fukagawa ${ }^{5} \cdot$ Toshikazu Ushijima $^{4} \cdot$ Hitoshi Katai $^{5} \cdot$ Ryo Wada $^{6} \cdot$ Koichi Sato $^{3} \cdot$ Kazuhiro Sakamoto $^{2}$. Wayne $\mathrm{Yu}^{7} \cdot$ Michael Considine $^{8} \cdot$ Leslie Cope $^{8} \cdot$ Malcolm V. Brock $^{1}$}

1 Department of Surgery, The Sidney Kimmel Comprehensive Cancer Center, Johns Hopkins University School of Medicine, 600N. Wolfe Street, Blalock 240, Baltimore, MD 21287, USA

2 Department of Coloproctological Surgery, Juntendo University Faculty of Medicine, Tokyo, Japan

3 Department of Surgery, Juntendo University Shizuoka Hospital, Shizuoka, Japan

4 Division of Epigenomics, National Cancer Center Research Institute, Tokyo, Japan
5 Gastric Surgery Division, National Cancer Center Hospital, Tokyo, Japan

6 Department of Pathology, Juntendo University Shizuoka Hospital, Shizuoka, Japan

7 Microarray Core Facility, Sidney Kimmel Comprehensive Cancer Center, School of Medicine, Johns Hopkins University, Baltimore, MD, USA

8 Experimental and Computational Genomics Core, Sidney Kimmel Comprehensive Cancer Center at Johns Hopkins, Baltimore, MD, USA 